\title{
COVID-19 PANDEMIC AND CHALLENGES IT POSES TO OTORHINOLARYNGOLOGISTS
}

\author{
V. Stoyanov* \\ Department of ORL, Trakia University, Stara Zagora, Bulgaria
}

\begin{abstract}
As doctors, we all have a shared responsibility in terms of COVID-19. At first glance, ENT specialists are not classed as the so-called "front line" medical workers but have a key role to play and these needs to be addressed. In response to the pressures on the Health Care System, the medical care provided by otorhinolaryngologists has been limited. Irrespective of the imposed work limitations, patients will still need to be provided with the specific care, which requires us to find the best solutions for work stations and wards.

In addition, measures should be put in place to avoid compromising surgical activity (especially emergency procedures), deriving from a combination of factors, including staff sickness, problems with the supply of medical equipment, the use of operating theaters, anesthesiological teams, etc.

An increased number of performed tracheostomies are expected in positive COVID-19 patients who need artificial pulmonary ventilation.

The purpose of this paper is to address all these issues, based on current and accessible information, and propose measures and procedures for otorhinolaryngologists in the context of the COVID-19 pandemic for different patient groups.

Simultaneously, recommendations are made to protect the doctors and their teams, as well as to reconfigure the medical operations in the new conditions.
\end{abstract}

Key words: ENT, COVID-19, tracheostomy, patient safety.

In December 2019, a series of patients with pneumonia caused by the novel coronavirus (COVID-19) emerged in Wuhan, Hubei, China (1). The rapid spread of the coronavirus epidemic has led to epochal changes in our daily habits, as well as changes in normal clinical and surgical practice. Since WHO officially announced on 12 March 2020 that COVID-19 infection is a real global pandemic (2), healthcare workers must always apply adequate infection control measures and must act to protect both patients and their own health as much as possible (3-5). We are witnessing decrease in the resources in healthcare, while the available ones are redirected to managing the pandemic, meaning equipment; PPE and workforce are considerably reduced in the routinely planned

*Correspondence to: Valentin Stoyanov, Dept. of ORL, Trakia University, Stara Zagora, 11 Armejska Str., Bulgaria,e-mail:valentin.stoyanov@trakiauni.bg clinical and surgical operations (6). As medical practitioners, we all have a responsibility toensure that the main concern for Bulgarian citizens' ENT health continues with a minimal pressure on Bulgarian National Healthcare System. ENT teams may need to work outside of their specific areas of expertise when undesirable exceptional circumstances arise as the epidemic develops. ENT specialists may not seem to be working on the front line with COVID-19, however, they have a key role to play in this process and this needs to be taken under consideration. In response to the pressures on the National Healthcare System, ENT operations capacity can be limited. Regardless of COVID-19, patients will continue to need care. The best solution for each specific ward or work station needs to be implemented in order to continue delivering correct treatment to these patients. These measures must be compliant with Government regulations and guidelines, the Rules of Good Medical Practice, the medical standard of ENT diseases and the established international 
standards in the field. The adopted solution must also observe maintaining a bank of resources for the response of COVID-19.

In addition, it needs to be considered how to avoid the disruption of surgical operations (especially emergency ones), due to a combination of factors including staff sickness, shortages in medical supplies and personal protective equipment, availability of theaters, availability of anesthesiologic teams, etc. (7) We may be expected to perform large number of tracheostomies in positive COVID-19 patients who need ventilation (8).

At the same time, some COVID-19-specific otorhinolaryngological symptoms should be detected (9), to be described, summarised and adopted in the relevant guidelines after careful expert discussions at national level.

The aim of this paper is to summarise current world wide studies on the approach to the pandemic and to make recommendations for procedures, as well as to outline some sectorspecific guidelines in the context of a pandemic.

\section{Personal protective equipment (PPE)}

Any healthcare professional working with patients with suspected or confirmed COVID19 must wear appropriate PPE.

Currently, the minimum requirement is to wear a fluid-resistant surgical mask, disposable apron, gloves, and eye protection (safety goggles and/or helmet). This applies to the diagnostic and operational treatment of ears, nose, throat, and esophagus and lower respiratory tract associated with the use of rigid or fibro-endoscopes. It should be noted that there are three main types of surgical face masks and not every type will offer complete protection to the ENT specialist and their team in the conditions of the COVID-19 pandemic (which is described in further details below). Konda and co-authors present a detailed discussion of the composition of the masks and note that gaps resulting from improper placement of the mask can lead to over $60 \%$ reduction in filtration efficiency. (10)

Particular attention should be paid when dealing with patients with confirmed (or suspected) COVID-19 who need a tracheostomy. On this issue, most authors argue that intubation is preferable, since tracheostomy is a high-risk procedure due to aerosol generation (11-14). However, if tracheostomy is inevitable, both Italian and Dutch professionals $(12,15,16)$ recommend the following stringent measures:

1. The number of members in the team engaged in carrying out the procedure shall be reduced to the possible minimum.

2. The highest qualified anesthetist and ENT specialist should perform the anesthesia and procedure to ensure that it is safe, accurate and fast.

3. All members in the team must use:

3.1. FFP3 mask;

3.2. Option for eyes/face protection when performing a tracheostomy or when replacing the tracheostomy cannula due to the risk of respiratory secretions or bodily fluids:

3.2.1 Surgical mask with integrated eye visor.

3.2.2 Full face shield/visor.

4. Use of disposable, moisture-resistant apron/scrubs. If the apron is not resistant to liquids, wear a disposable nylon scrub underneath.

5. Gloves should be suitable to allow palpation, the use of sutures and surgical instruments, but necessarily use the "Eclipse system" or double layering of gloves.

6. If possible, change the tracheostomy tube only after COVID-19 had run its course.

Personal protective equipment is only a part of the measures for preventing crosscontamination and infections in healthcare workers. In addition to them, procedures such as disinfecting surfaces and equipment and waste management are essential to reduce risk. $(15,17)$.

The use of reliable PPE has been shown to have reduced the risk of infections among healthcare workers during the SARS epidemic (16) and we should apply the knowledge from this experience now.

Operational procedures when admitting assistance.

In general, this group of patients can be grouped into several categories:

1. Mandatory emergency inpatients.

Patients can be admitted in a hospital for surgical intervention only when there is a clear obstruction to the respiratory tract (foreign object in the tract, Oedema Quincke, etc.). 
Emergency cases should be treated as early as possible in order to prevent further complications, including reanimation and/or treatment in ICU (Intensive Care Unit). At the same time, correct procedures must be observed when working with endoscopic equipment in theatre, isolated from the other wards.

2. Non-surgical emergencies.

This group is relatively less common in practice, with the highest percentage of cases represented by nosebleeds (epistaxis). These patients can be treated in a non-surgical manner, and the aim is to avoid hospital admission, unless this is imperative.

3. Scheduled admissions.

These must be prioritised during current conditions. With non-surgical cases being postponed, scheduled admissions are the only acceptable hospitalisations. This approach may also protect the patients from longer exposure to the risks during a stay in a hospital, while also freeing up beds for urgent cases.

4. Single-day surgery.

Most planned ENT surgeries can safely be performed within a day. When conditions are not met to perform a single day procedure, efforts should be made to create appropriate conditions, as inpatient surgery should be closed for such activities. Priority in the conditions for a single day surgery is those allowing local anesthesia to complete the procedure. During the COVID-19 crisis, the increase in single day surgeries helps to:

$\sqrt{ }$ avoid unnecessary admissions

$\checkmark$ decrease patient exposure to certain

infections in a hospital environment

$\checkmark$ free up hospital beds for urgent cases

$\checkmark$ allows healthcare practitioners from relevant departments/operating rooms to continue working in a familiar environment.

\section{Operational procedures for specialised outpatient care}

Scheduled outpatient visits should be reduced to an acceptable minim. Most routine ENT outpatient checks may be safely postponed. Telephone consultations must be implemented where possible, especially with vulnerable groups of patients. Using internet-based applications and methodology from telemedicine should also be adopted to minimise admitting outpatients. These steps should help to decrease the pressure in Emergency Wards which are popular with patients with minor ENT health issues. When it is inevitable to see a patient, the following rules must be strictly observed:

1. Manage appointments so no more than 2 patients will be in a waiting room at any one time.

2. Doctors and hospital staff must always wear PPE; patients must wear face masks.

3. If the patient is a child or a vulnerable person, they can be allowed only one companion.

4. Mandatory disinfection of surfaces shall be undertaken after seeing each patient, including door handles, toilet bowls, etc.

5. Hand sanitiser station at the entrance Outpatient care must be provided for the patient to use upon entering the building/facility.

\section{CONCLUSIONS}

1. Carrying out specialized ENT activities in both hospital and outpatient care must be completely reconfigured and reorganised in the context of COVID-19 pandemic.

2. It is essential to protect the lives and health of doctors and other medical staff in order to respond adequately to the pressures on the healthcare system caused by the pandemic.

3. Personal protective equipment is of paramount importance for the successful preservation of human resources.

4. Patients in need of urgent specialised ENT care must be taken over by highly experienced staff with knowledge in the area of COVID-19 procedures. The course of treatment for each patient must be agreed by a multidisciplinary team comprising of various specialists depending on the case - anesthesiologist, pediatrician, oncologist, etc.

As a result of the overview above, we could add the following recommendations to the outlined operational procedures:

1. Avoid unwanted/unnecessary hospital visits.

1. Terminate planning operations.

2. No patient should be scheduled for surgery without preliminary discussion by a multidisciplinary team of experts.

3. Introduce an emergency ENT cabinet, operating for patients from 9:00 to 17:00 (if possible, with longer working hours), to reduce the pressures on emergency departments.

4. Postpone scheduled appointments (when possible!) and long-term monitoring of patients until the pandemic passes. The use 
of telephone consultations in these cases is a viable option.

\section{REFERENCES}

1. Huang, C., Wang, Y., Li., et al., Clinical features of patients infected with 2019 novel coronavirus in Wuhan, China. Lancet. 395: 497-506, 2020. doi: 10.1016/S0140-6736(20)30183-5.

2. World Health Organization. Coronavirus disease 2019 (COVID-19) Situation Report - 51. Available

from: https://www.who.int/docs/default-

source/coronaviruse/situation-

reports/20200311-sitrep-51-covid-

19.pdf?sfvrsn=1ba62e57_10.

3. Adalja, A., Toner, E., Inglesby, V., Priorities for the US health community responding to COVID-19. JAMA, 2020. doi:10.1001/jama.2020.3413.

4. Spina, S., Marrazzo, F., Migliari, M., Stucchi, R., Sforza, A., Fumagalli, R., The response of Milan's emergency medical system to the COVID-19 outbreak in Italy, Lancet North Am Ed, 2020. 395(10227): e49-e50.

5. Burke, F., Chan, K., Mummaneni, V., Chou, D., Lobo, P., Berger S., et al., Letter: The Coronavirus Disease 2019 Global Pandemic: A Neurosurgical Treatment Algorithm. Neurosurgery, 2020. pii: nyaa116. https://doi.org/10.1093/neuros/ny aa116.

6. Zheng L., Yawei Z., Xishan W., et al., Recommendations for Surgery During the Novel Coronavirus (COVID-19) Epidemic. Indian J Surg, 11: 1-5, 2020. doi: 10.1007/s12262-020-02173-3 [Epub ahead of print] PMCID: PMC7150607 PMID: 32292252.

7. Brindle, M., Gawande, A., Managing COVID-19 in Surgical Systems, Ann Surg, 2020.

https://doi.org/10.1097/SLA.000000000000 3923.

8. Tay, K., Khoo, L., Loh, S., Surgical Considerations for Tracheostomy During the COVID-19 Pandemic: Lessons Learned From the Severe Acute Respiratory Syndrome Outbreak, JAMA Otolaryngol Head Neck Surg, 2020. https://doi.org/10.1001/jamaoto.2020. 0764 .
9. Carol, Y., F, Faraji., Divya, P., Boone, C., DeConde, A,. Association of chemosensory dysfunction and Covid-19 in patients presenting with influenza-like symptoms. International Forum of Allergy \& Rhinology, 2020; DOI: 10.1002/alr.22579.

10.Konda, A., et al., Aerosol filtration efficiency of common fabrics used in respiratory cloth masks. ACS Nano Article ASAP DOI: $10.1021 /$ acsnano.0c03252.

11.ENT UK Guidance for ENT during the COVID-19 pandemic.

12.https://www.entuk.org/tracheostomyguidance-during-covid-19-pandemic-1harrison-et-al

13.http://www.attingoedu.it/en/recommendations-covid-19-profcastelnuovo-sib.html

14.Tran, K., Cimon, K., Severn, M., PessoaSilva, C., Conly, J., Aerosol generating procedures and risk of transmission of acute respiratory infections to healthcare workers: a systematic review. PLoS One, 2012, 7: e35797.

15.van Doremalen, N., Bushmaker, T., Morris, D., et al., Aerosol and surface stability of HCoV-19 (SARS-CoV-2) compared to SARS-CoV-1. NEJM in press doi:https://doi.org/10.1101/20

20.03.09.20033217.https://www.medrxiv.or $\mathrm{g} /$ content/10.1101/2020.03.09.20033217v1. full.pdf.

16.Loeb, M., McGeer, A., Henry, B., et al., SARS among critical care nurses. Emerg Infect Dis; 10: 251-5, 2004.

17.Public Health England. Environmental decontamination, in COVID-19: infection prevention and control guidance https://www.gov.uk/government/publicatio ns/wuhan-novel-coronavirus-infectionprevention-and-control/wuhan-novelcoronavirus-wn-cov-infection-preventionand-control-guidance\#decon.

18.COVID-19 Guidance for Infection Prevention and Control in Healthcare Settings. $\quad$ Version 1.0 https://www.gov.uk/government/publicatio ns/wuhan-novel-coronavirus-infectionprevention-and-control. 